education and were behaviorally impaired. (Autti-Ramo I. Dev Med Child Neurol June 2000;42:406-411). Follow-up should be focussed not only on the child's cognitive and neuromotor development but on parental and foster family support and counseling and the prevention of secondary behavioral problems.

\title{
AMPHETAMINE ABUSE AND INTRACRANIAL HEMORRHAGE
}

Eight young adults presenting with intracranial hemorrhage at the Department of Neurosurgery, Queen's Medical Center and University Hospital, Nottingham, UK, in a 3 and $1 / 2$ year period, were diagnosed with amphetamine abuse. The time from amphetamine exposure to onset of symptoms ranged from 10 minutes to 2 months (median within 24 hours). CT and cerebral digital subtraction angiography (DSA) showed a parenchymal hematoma in 7 (3 in the frontal lobe), and a subarachnoid hemorrhage in 1. Beading of small and medium-sized arteries, characteristic of vasculitis, also occurred in 1. Four recovered, but 1 died and 3 were hemiplegic.

Of 37 previously reported cases, from 1945 to 1996,6 died and 17 had hemiparesis. One-third claimed to be infrequent amphetamine users. The first reported death from amphetamine abuse (IAMA 1939) was in a 25 year-old man who had taken the drug as a stimulant before a college examination. Amphetamine use or abuse should be considered in diagnosis of a frontal or parietal lobe hematoma in a young patient. (Buxton N, McConachie NS. Amphetamine abuse and intracranial hemorrhage. LR Soc Med September 2000;93:472-477). (Respond: Mr Neil Buxton FRCS (Ed), Department of Neurosurgery, University Hospital, Queen's Medical Centre, Nottingham NG7 2UH, UK).

COMMENT. Amphetamines taken orally, IV, or rarely, inhaled, have resulted in cerebral vasculitis and intracranial hemorrhage. Unfortunately, the dosages involved are unknown. The outcome is poor in more than half the cases reported. Vasculitis, that can follow only a single exposure to oral amphetamines, is characterized by irregular segmental narrowing or beading of small cerebral arteries.

Perhaps the recent increased popularity of amphetamine drugs in the treatment of ADHD in school-age children should be reappraised.

\section{CELIAC ANTIBODIES AND NEUROLOGIC DISORDERS}

The yield of screening for celiac disease in children with common neurologic disorders was evaluated at Tel Aviv University, Zerifin, Israel. Of 167 patients presenting, ages 1 to 16 years, 41 had migraine headaches, $39 \mathrm{ADHD}, 36$ epilepsy, and 51 hypotonia and motor abnormalities. IgG antigliadin antibodies were positive in 22 (13\%) of patients compared to $3(9 \%)$ in the control group. IgA and endomysial antibodies were negative in all patients, and duodenal biopsies were not performed. Routine screening for celiac disease is not recommended in children presenting wsith common neurologic disorders. (Lahat E, Broide E, Leshem M, Evans S, Scapa E. Prevalence of celiac antibodies in children with neurologic disorders. Pediatr Neurol May 2000;22:393-396). (Respond: Dr Eli Lahat, Unit of Pediatric Neurology, Assaf Harofeh Medical Center, Zerifin 70300, Israel).

COMMON. Neurologic complications of celiac disease include peripheral neuropathy, myopathy, cerebellar ataxia, myoclonus, cerebral atrophy, cerebral vasculitis, encephalitis, epilepsy, sometimes associated with cerebral calcification. Studies in adults have shown a significant increase in positive titers for antigliadin antibodies in patients (57\%) with common neurologic disorders of undetermined etiology. In children, the association of celiac disease with common 
neurologic disorders is apparently less remarkable. However, in patients with ataxia, myopathy, or seizures of undetermined etiology, a test for antigliadin antibodies should be considered, especially as symptoms may be reversible with a gluten-free diet.

\section{NEUROECTODERMAL DISORDERS}

\section{NEUROFIBROMATOSIS 1 AND GROWTH HORMONE DEFICIENCY}

The growth profiles and growth hormone levels of children with neurofibromatosis 1 (NF-1) were analyzed at the University of Texas Medical School, Houston, TX. Of 251 children attending the clinic with NF-1, 112 were at or below the 25th percentile for height. Of these, 51 were showing poor growth rates, without radiological evidence of suprasellar lesions. Growth hormone deficiency (GHD), with peak GH levels of less than $5 \mathrm{ng} / \mathrm{ml}$, was determined in 15 of 19 tested. (Vassilopoulou-Sellin R, Klein MJ, Slopis JK. Growth hormone deficiency in children with neurofibromatosis type 1 without suprasellar lesions. Pediatr Neurol May 2000;22:355-358). (Respond: Dr Vassilopoulou-Sellin, Section of Endocrinology, Box 15, University of Texas, Anderson Cancer Center, 1515 Holcombe Blvd, Houston, TX 77030).

COMMENT. Growth hormone deficiency and short stature may be associated with neurofibromatosis 1 , independent of organic pituitary lesions.

\section{TOURETTE'S SYNDROME AND NEUROFIBROMATOSIS 1}

A case of Tourette's syndrome in association with neurofibromatosis 1 is reported in an 11-year-old boy evaluated at the Instituto de Ciencias Neurologicas, Lima, Peru. Tics began at 5 years, manifested by excessive blinking, and followed by head nodding and shoulder shrugging. At 6 years of age, he developed symptoms of ADHD and learning problems, leading to academic underachievement. At 8 years of age, tic vocalizations began, and at 10 years, a painless intranasal tumor developed that caused deformity of the nasal cartilage. Examination revealed multiple cafe-au-lait spots. Biopsy of the nasal tumor revealed a neurofibroma. MRI showed hyperintensities (T2-weighted) and hypointensities (T1-weighted) in both pallidi. Head CT was normal. (Cosentino C, Torres L. Tourette's syndrome and neurofibromatosis 1. Pediatr Neurol May 2000;22:420-421). (Respond: Dr Carlos Cosentino, Instituto de Ciencias Neurologicas, Ancash 1271, Lima 1, Peru).

COMMENT. This may be the first documented case of an association between neurofibromatosis 1 and Tourette's syndrome.

Hemolytic streptococcal infection as a precipitant of tics $w$ as evaluated at Johns Hopkins University, Baltimore, MD (Singer HS, Giuliano JD, Zimmerman AM, Walkup JT. Pediatr Neurol May 2000;22:3809-383). Of 80 consecutive children with a diagnosis of tic disorder, $53 \%$ had a sudden onset or worsening of tics, and in 15 of these 42, the exacerbation was associated with infection, streptococcal in 9 . Approximately $11 \%$ of children with tic disorders have a worsening of symptoms within 6 weeks after a streptococcal infection. A relationship of strep infection to tic disorders continues to unfold. 